\title{
In silico analysis of promoter regions and regulatory elements (motifs and CpG islands) of the genes encoding for alcohol production in Saccharomyces cerevisiaea S288C and Schizosaccharomyces pombe 972h-
}

Jemal Aman Beshir ${ }^{1,2^{*}}$ (D) and Mulugeta Kebede ${ }^{1}$

\begin{abstract}
Background: The crucial factor in the production of bio-fuels is the choice of potent microorganisms used in fermentation processes. Despite the evolving trend of using bacteria, yeast is still the primary choice for fermentation. Molecular characterization of many genes from baker's yeast (Saccharomyces cerevisiaea), and fission yeast (Schizosaccharomyces pombe), have improved our understanding in gene structure and the regulation of its expression. This in silico study was done with the aim of analyzing the promoter regions, transcription start site (TSS), and CpG islands of genes encoding for alcohol production in S. cerevisiaea S288C and S. pombe 972h-.

Results: The analysis revealed the highest promoter prediction scores (1.0) were obtained in five sequences (AAD4, SFA1, GRE3, YKL071W, and YPR127W) for S. cerevisiaea S288C TSS while the lowest (0.8) were found in three sequences (AAD6, ADH5, and BDH2). Similarly, in S. pombe 972h-, the highest (0.99) and lowest (0.88) prediction scores were obtained in five (Adh1, SPBC8E4.04, SPBC215.11C, SPAP32A8.02, and SPAC19G12.09) and one (erg27) sequences, respectively. Determination of common motifs revealed that S. cerevisiaea S288C had 100\% coverage at MSC1 with an E value of 3.7e-007 while S. pombe $972 h$ - had $95.23 \%$ at MSp1 with an E value of $2.6 \mathrm{e}+002$. Furthermore, comparison of identified transcription factor proteins indicated that $88.88 \%$ of MSp1 were exactly similar to MSC1. It also revealed that only $21.73 \%$ in S. cerevisiaea S288C and $28 \%$ in S. pombe $972 \mathrm{~h}$ - of the gene body regions had $\mathrm{CpG}$ islands. A combined phylogenetic analysis indicated that all sequences from both $S$. cerevisiaea S288C and S. pombe 972h- were divided into four subgroups (I, II, III, and IV). The four clades are respectively colored in blue, red, green, and violet.

Conclusion: This in silico analysis of gene promoter regions and transcription factors through the actions of regulatory structure such as motifs and CpG islands of genes encoding alcohol production could be used to predict gene expression profiles in yeast species.
\end{abstract}

Keywords: Alcohol production, CpG islands, Motifs, Saccharomyces cerevisiaea S288C, Schizosaccharomyces pombe 972h-

\footnotetext{
* Correspondence: jemalbeshir@gmail.com

'Department of Applied Biology, School of Applied Natural Science, Adama

Science and Technology University, P.O. Box 1888, Adama, Ethiopia

${ }^{2}$ Ethiopian Sugar Corporation, Sugar Academy, Wonji, Ethiopia
}

\section{Springer Open}

(- The Author(s). 2021 Open Access This article is licensed under a Creative Commons Attribution 4.0 International License, which permits use, sharing, adaptation, distribution and reproduction in any medium or format, as long as you give appropriate credit to the original author(s) and the source, provide a link to the Creative Commons licence, and indicate if changes were made. The images or other third party material in this article are included in the article's Creative Commons licence, unless indicated otherwise in a credit line to the material. If material is not included in the article's Creative Commons licence and your intended use is not permitted by statutory regulation or exceeds the permitted use, you will need to obtain permission directly from the copyright holder. To view a copy of this licence, visit http://creativecommons.org/licenses/by/4.0/. 


\section{Background}

The scarcity and rising prices of fossil fuels, geo-political instability in countries that hold most of the proven oil reserves together with apprehension about the environmental harm created by them, have resulted in increasing efforts to search for alternative energy sources [1]. Hence, production and use of bio-fuels for transport fuel has recently attracted significant attention worldwide. Likewise, Ethiopia's sustainable development and the national fuel security can only be realized with increased production and utilization of renewable fuels. Substituting the demand for fossil fuel by locally produced fuels such as bio-ethanol and bio-diesel is paramount importance for the country's economic use of scarce energy resources.

Bio-ethanol has been made since ancient times by fermenting sugars, and most bio-ethanol used for fuel and alcoholic drinks, and most industrial ethanol, is made by this process (Licht 2001; as cited in [2]). Great strides in research together with the development of new yeast strains have led to demands to model a new yeast strain which can withstand and produce at higher levels of alcohol, temperatures, and $\mathrm{pH}$. This requires immense knowledge of the fermentation processes to improve its efficiency which is dependent on various factors, namely, process design, molasses quality, yeast strain, contamination, nutrient availability, and raw material purity [2]. Yeast alcohol is one of the most valuable products originating from the biotechnological industry with respect to both value and amount [3]. Yeast selection for fuel ethanol production over the past two decades and most bio-ethanol-related researches in developing tropical countries have focused primarily on the isolation of local Saccharomyces yeasts and their use for industrial ethanol production [4-6].

The development of DNA transformation in yeast has made possible the rapid molecular isolation of many genes from baker's yeast (Saccharomyces cerevisiaea) and fission yeast (Schizosaccharomyces pombe). Concomitantly, our understanding of many aspects of gene structure and regulation of gene expression in these organisms has improved. Although the two organisms are similar in that they are both spore-forming yeasts capable of strong alcoholic fermentation, S. pombe actually has diverged significantly from S. cerevisiaea [7]. In recent years, genome mining and in silico analysis of gene sequences and their products have become a key methodology to identify gene expression patterns, sequences responsible for development of new molecules, leading to the discovery of dozens of novel compounds $[8,9]$. A variety of computational tools have been developed to support scientists in this field. Most of the available tools are dedicated to the in silico analysis of specific gene and gene products [8].
Gene expression varies among tissues and even different cell types but also in response to specific signals (physiological, environmental, etc.). The main mechanism of transcriptional regulation is orchestrated by proteins called transcription factors (TFs), which promote (as activators) or block (as repressors) the recruitment of the RNA polymerase II (Pol II complex) [10]. The promoter is a DNA sequence that the transcription apparatus recognizes and binds. It indicates which of the two DNA strands is to be read as the template and the direction of transcription [11]. It is a functional region containing complex regulatory elements for determining the transcription initiation of genes $[9,10,12,13]$. DNAbinding sites or motifs refer to short DNA sequences (typically 4 to 30 base pairs long, but up to $200 \mathrm{bp}$ for recombination sites) that are explicitly bound by one or more DNA-binding proteins or protein complexes [14]. It is often associated with specialized proteins known as transcription factors and is thus linked to transcriptional regulation. A structural feature that has proven useful in the detection of promoters is the so called CpG islands, i.e., regions that are rich in CpGs, which are important because of their strong link with gene regulation[15]. $\mathrm{CpG}$ islands are playing an important role in gene regulation through epigenetic changes [16].

Therefore, the aim of this study is to predict promoter and regulatory elements of genes encoding alcohol production in yeast species (Saccharomyces cerevisiaea S288C and Schizosaccharomyces pombe $972 h$-) thereby providing basic information which could support the effort of improving them for a commercial-scale bio-ethanol production.

\section{Methods}

Determination of transcription start sites and promoter regions for genes encoding alcohol production

Gene sequences of yeast species (Saccharomyces cerevisiaea S288C and Schizosaccharomyces pombe 972h-) for genes encoding alcohol production were retrieved as FASTA file from NCBI Genome Browser (https://www. ncbi.nlm.nih.gov/gene). Gene sequences starting by ATG (starting codon) were identified, and coding sequences were used in this analysis. However, for S. pombe 972h-, all the sequences retrieved from direct NCBI web were not having the functional gene structure (no ATG in the beginning and many stop codons in the middle). Therefore, sequences used for the current study were retrieved via NCBI Reference Sequences (RefSeq). This section includes genomic Reference Sequences (RefSeqs) from all assemblies on which these genes were annotated, such as RefSeqs for chromosomes and scaffolds (contigs) from both reference and alternate assemblies. To this end, gene sequences were taken as identical protein annotated from PomBase annotation Provider for Eukaryotic Annotation Propagation Pipeline. 
Generally, 23 and 21 sequences of alcohol dehydrogenase were retrieved for S. cerevisiaea S288C and S. pombe $972 h$-, respectively. To determine respective transcriptional start sites (TSSs) for all gene sequences, about 1$\mathrm{kb}$ sequences upstream of the start codon were excised from all genes except for ADH1 of S. cerevisiaea S288C which was at 2-kb upstream of start codon. Similarly, all gene sequences of $S$. pombe $972 \mathrm{~h}$-, except three genes (erg27 at $2.5 \mathrm{~kb}$ and SPBC8E4.04 and SPBC337.11 at 2 $\mathrm{kb})$, had TSSs at 1-kb sequences upstream of their start codons.

The Neural Network Promoter Prediction (NNPP version 2.2) tool set was used with the minimum standard predictive score (between 0 and 1) [17]. For those regions containing more than one TSS, the one with the highest value of prediction score was considered to have trustable and truthful prediction. Promoter regions were defined as 1-kb region upstream of each TSS. For those regions containing more than one TSS, the highest value of prediction score will be considered so as to have a more accurate prediction.

\section{Determination of common motifs and TFs for genes encoding alcohol production in the promoter region} Analysis of conserved motifs for genes encoding for alcohol productions for both yeast species was performed by MEME (Multiple Em for Motif Elicitation) software version 3.5.4 (http://meme.sdsc.edu). This online webbased analysis was performed with minimum and maximum motif width of 6 and 50 residues, respectively, for both yeast species whereas a maximum number of motifs for S. cerevisiaea S288C and S. pombe $972 \mathrm{~h}$ - were 23 and 21 , respectively, which were used to identify probable promoter regulatory elements (motifs), keeping the rest of the parameters at default. The MEME output in HTML showed the motifs as local multiple alignments of the input sequences, as well as in several other formats. Buttons on the MEME HTML output were allowed one or all of the motifs to be forwarded for additional investigation. Descriptions the identification of motifs by TOMTOM [18] web server were designated where numerous sequence databases can be searched for sequences matching the identified motif, in which the output of TOMTOM will include LOGOS on behalf of the alignment of two motifs, the $p$ value and $q$ value (a measure of false discovery rate) of the match [18]. TOMTOM showed that the query motif closely resembles the binding motif in the set of genes encoding for alcohol production promoter regions.

\section{Search for CpG islands for genes encoding alcohol production promoter regions}

To search CpG islands, first, the stringent search criteria were used in the Takai and Jones algorithm: GC content $\geq 50 \%$, Obs CpG/ExpCpG $\geq 0.60$, and length $\geq 200 \mathrm{bp}$ [20]. For this purpose, the CpG island searcher program (CpGi130) available at web link (http://www.bioinformatics.org/sms/cpg_island.html) was used. The CpG island graphs were plotted using EMBOSS Cpgplot (https://www.ebi.ac.uk/Tools/seqstats/emboss_cpgplot/) which identify and plot $\mathrm{CpG}$ islands in nucleotide sequence(s). Secondly, the CLC Genomics Workbench ver. 3.6.5 (http://clcbio.com, CLC bio, Aarhus, Denmark) was used for restriction enzyme MSpI cutting sites (fragment sizes between 40 and $220 \mathrm{bp}$ ).

\section{Phylogenetic analysis}

Phylogenetic analysis of gene sequences of both yeast species (S. cerevisiaea S288C and S. pombe 972h-) was conducted using the Molecular Evolution Genetic Analysis 6 (MEGA6) tool by the neighbor-joining treemaking method. Similarly, Tajima's neutrality test of selection was conducted using the same software to find nucleotide diversity. The p-distance model was applied with transition and trans-version nucleotide substitution. Bootstrap values of the super tree were computed with 2000 repetitions with uniform rate among sites and complete deletion of gaps/missing data were used to analyze the sequences.

\section{Results}

Determination of transcription start sites and promoter regions for genes encoding alcohol production

Promoter region analysis of genes encoding for alcohol productions of both yeast species (S. cerevisiaea S288C and S. pombe 972h-) showed a great variation in the number of TSS. The highest promoter prediction scores (1.0) for TSS of S. cerevisiaea S288C alcohol dehydrogenase were obtained for five gene sequences (AAD4, SFA1, GRE3, YKL071W, andYPR127W) while the lowest promoter prediction scores $(0.8)$ were obtained for three gene sequences (AAD6, ADH5, and BDH2) (Table 1). In addition, the result of promoter predictions for $S$. cerevisiaea S288C sequences with score cutoff 0.80 showed that out of twenty-three gene sequences used in this analysis only ADH1 and ADH7 (8.70\%) had showed a single TSS while the remaining (91.30\%) showed multiple TSS. In these scenarios, TSSs with the highest prediction scores were considered for further uses. TSSs of genes encoding for alcohol production in S. cerevisiaea S288C were mostly located in the upstream region of 31 to $-1545 \mathrm{bp}$, with the relatively highest incidence of occurrence in the upstream region of -1 to $-200 \mathrm{bp}$ (10 sequences, $43 \%$ ) followed by -201 to $-400 \mathrm{bp}$ and 601 to $-800 \mathrm{bp}$ (4 sequences each, 17.4\%) from the transcription start site, while the lowest occurrence was observed at -801 to $-1000 \mathrm{bp}$ and above $-1000 \mathrm{bp}$ (only 1 sequence each). 


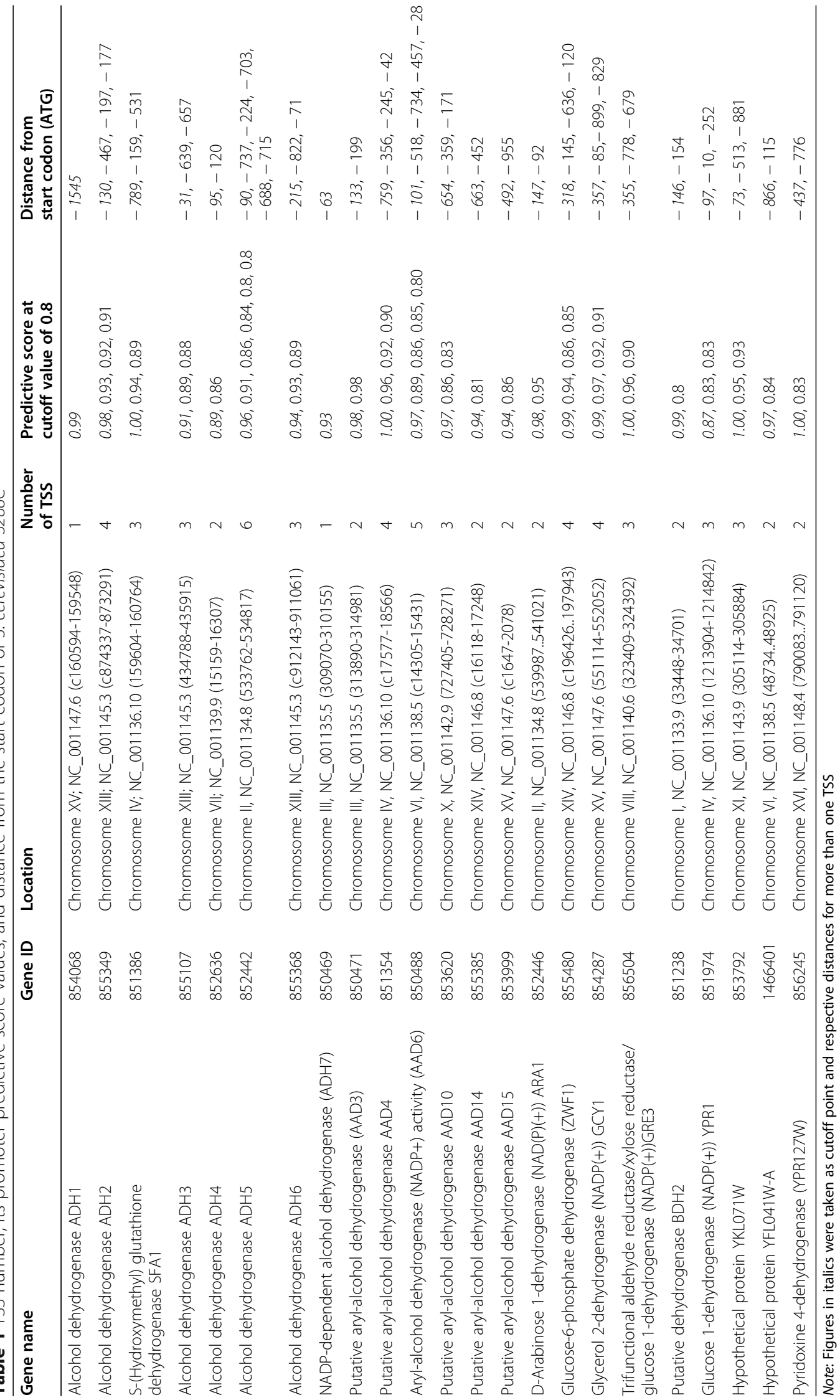




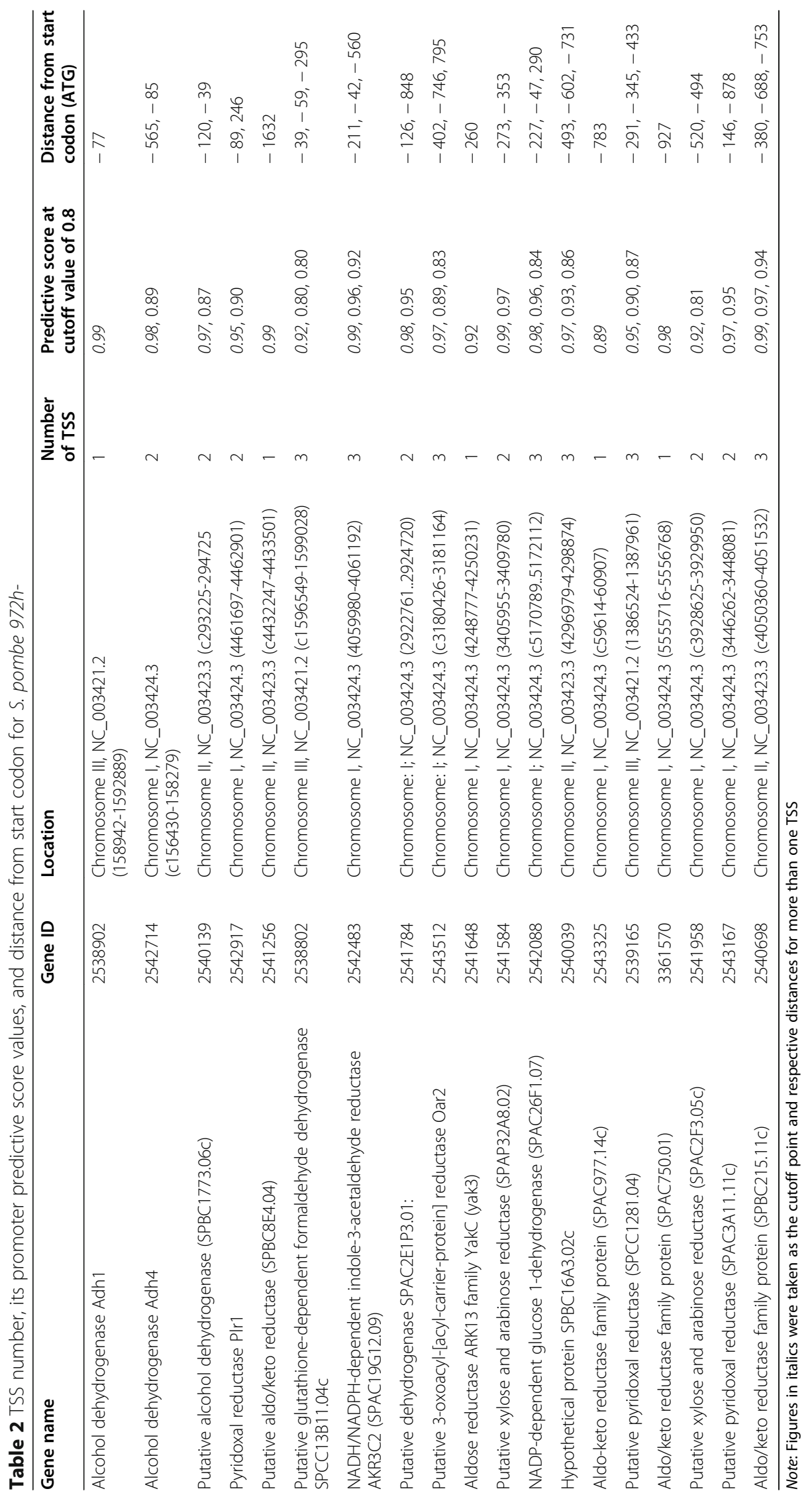


Table 3 List of discovered motifs and the number and proportion of promoter-containing motifs for S. cerevisiaea S288C

\begin{tabular}{lllll}
\hline Discovered motif & Number (\%) of promoter-containing motifs & E value & Motif width & Total number of binding sites \\
\hline MSC1 & $23(100 \%)$ & $3.7 e-007$ & 15 & 23 \\
MSC2 & $18(78.26 \%)$ & $1.8 e+004$ & 29 & 18 \\
MSC3 & $8(34.78 \%)$ & $2.5 e+004$ & 10 & 8 \\
MSC4 & $3(13.04 \%)$ & $4.8 e+004$ & 29 & 3 \\
MSC5 & $7(30.43 \%)$ & $1.6 e+004$ & 29 & 7 \\
\hline
\end{tabular}

MSc motif for S. cerevisiaea S288C

Likewise, twenty-one sequences of genes encoding for alcohol production for $S$. pombe $972 h$ - (fission yeast) were retrieved from NCBI Genome Browser. Accordingly, the result of TSS and promoter analysis had showed a significant variation in the number of TSS and the distance of TSS from the start codons (Table 2). The numbers of TSSs were varied from 1 to 3 with majority of sequences $(71.43 \%)$ having more than one TSS. In particular to this fact, out of twenty-one sequences six, seven, and eight sequences had one, two, and three TSSs respectively. The relative locations of all TSS with respect to start codon were given in Table 2. The nearest TSS were recorded for SPCC13B11.04c (-39) followed by Adh1 ( -77$)$ while the far-flanged TSS were observed for egr27 (-2308) followed by SPBC337.11 (-1636) upstream of the start codons of their respective genes. The current analysis also revealed that the relatively highest frequency of occurrence in the upstream region of -1 to $-200 \mathrm{bp}$ and -201 to $-400 \mathrm{bp}$ (6 sequences each, $57.1 \%$ share) followed by -401 to -600 bp (4 sequences, $19.04 \%$ ) from the transcription start site, while the lowest occurrences were observed at -601 to -800 and 801 to -1000 bp (only 1 sequence each) and whereas three sequences had their TSS at above -1000 bp (Table 1 ). The predictive score at a cutoff value of 0.8 ranged from 0.99 to 0.88 .

\section{Determination of common motifs and TFs for genes} encoding alcohol production in the promoter region The promoter regions shared by majority of the gene sequences used in the current study revealed that $S$. cerevisiaea $S 288 C$ had $100 \%$ coverage among the gene sequences at $\mathrm{MSc} 1$ with an $E$ value of $3.7 \mathrm{e}-007$ and 15 motif widths (Table 3).

To determine motifs which are functionally important, motifs which were shared by majority of promoter regions of S. cerevisiaea $S 288$ C genes encoding for alcohol production were chosen. Accordingly, MSc1 was revealed as the common promoter motif for all (100\%) genes that serves as binding sites for transcription factors involved in the expression regulation of these genes. Sequence logo for MSc1 generated by MEME is presented in Fig. 1

Likewise, S. pombe $972 \mathrm{~h}$ - promoter sequences had 95.23\% conserved motif at MSp 1 with $E$ value of $2.6 \mathrm{e}+$ 002 and 29 motif widths (Table 4). The common motifs of MSp 1 shared by majority of the genes encoding for alcohol production sequences of S. pombe $972 h$ - as generated by MEME revealed in Fig. 2.

Furthermore, candidate transcription factor proteins were identified for motifs ( $\mathrm{M} S c 1$ and $\mathrm{MSp} 1$ ) of both yeast species. They were then compared to the registered motifs in publicly available database of Jaspar2018_ Core_Fungi_Non-Redundant DNA so as to see if they are similar to known regulatory motifs using TOMTOM web application [19]. The output from TOMTOM also links back to the parent motif database for more detailed information on biological functions of the matched motif. As a result, 13 motifs out of 176 common promoter motif/transcription factors were identified for $\mathrm{MSc} 1$ while only 9 motifs out of 176 in MSp 1 were being found matched with known motifs found in JASPAR

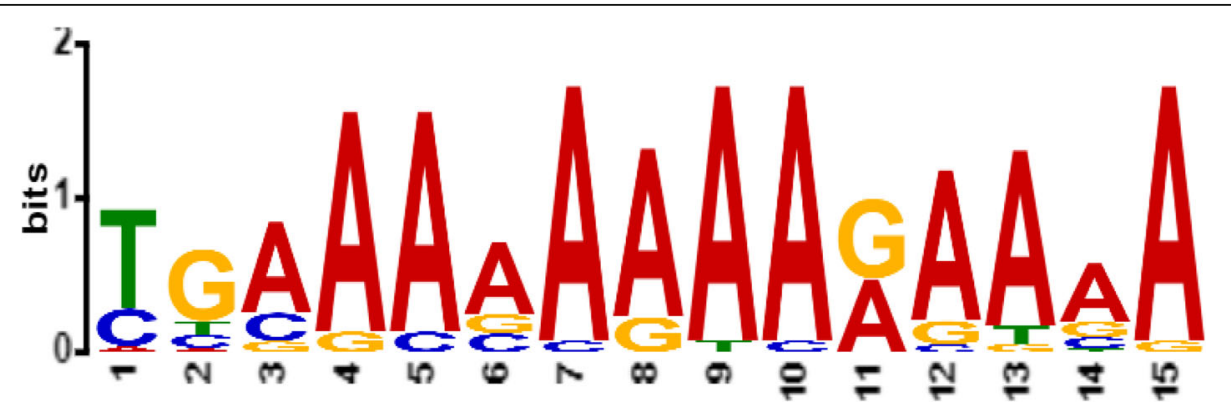

Fig. 1 Sequence logo for the identified common promoter motif MSC1 gene for gene encoding alcohol production gene in S. cerevisiaea S288C 
Table 4 List of discovered motifs and the number and proportion of promoter-containing motifs for S. pombe $972 h$ -

\begin{tabular}{lllll}
\hline Discovered motif & Number (\%) of promoter-containing motifs & E value & Motif width & Total number of binding sites \\
\hline MSp1 & $20(95.23 \%)$ & $2.6 e+002$ & 29 & 20 \\
MSp2 & $4(19.04 \%)$ & $1.5 e+004$ & 48 & 4 \\
MSp3 & $13(61.90 \%)$ & $1.8 \mathrm{e}+004$ & 15 & 13 \\
MSp4 & $6(28.57 \%)$ & $2.2 \mathrm{e}+004$ & 21 & 6 \\
MSp5 & $5(23.81 \%)$ & $1.8 \mathrm{e}+005$ & 19 & 5 \\
\hline
\end{tabular}

MSp motif for S. pombe

2018 CORE fungi motif databases (Table 2). In addition, the comparison of the identified transcription factor proteins indicated that almost $88.88 \%$ (8 out of 9) of the MSp1 were exactly similar to that of $M S c 1$. This could be due to the fact that these two yeast species ( $S$. cerevisiaea $S 288 \mathrm{C}$ and S. pombe $972 \mathrm{~h}$-) shared common promoters and their subsequent transcription factor protein were highly conserved regions of the genes encoding for alcohol production. TOMTOM is a motif comparison algorithm that ranks the target motifs in a given database according to the estimated statistical significance of the match between the query and the target. In similar manner, TOMTOM provides LOGOS that represents the alignment of two motifs and a numeric score for the match between two motifs together with a statistical significance [21].

The total numbers of motifs discovered in $S$. cerevisiaea $S 288$ C for genes encoding alcohol production promoter regions were about 60 out of which relatively, higher distributions of motifs were found also in positive (39) than in negative (21) strands (Fig. 3). The location and distribution of these motifs were ranged from -998 to -1 while higher concentration of motifs was found between -850 and -50 bp of the transcription start sites (TSSs). In the same view, only 48 motifs were discovered in S. pombe $972 h$ - out of which relatively, higher distributions of motifs were found also in negative (25) than in positive (23) strands (Fig. 4). The location and distribution of these motifs were ranged from - 996 to -2 while higher concentration of motifs was found between -800 and -100 bp of the transcription start sites (TSSs).

\section{Search for CpG islands for genes encoding alcohol production in the promoter regions}

In the current study, revealed $\mathrm{CpG}$ islands were examined in the promoter and gene body regions of both yeast species using both Takai and Jones algorithm using parameters as indicated in this section of this study and CLC Genomics Workbench ver. 3.6.5. Accordingly, as per the stringent criteria of Takai and Jones [17] as indicated in this section, there were only five (ADH1 (Fig. 5), ADH2, ADH5, ZWF1, and BDH2) (21.73\%) CpG islands observed in the gene body regions in analogous to only six (ADH1, SFA1, ADH3, ZWF1, BDH2, and YPR127W) out of twenty-three (26.08\%) gene sequences used for the analysis in promoter regions of $S$. Cerevisiaea $S 288 C$ yeast species. Likewise, only one (adh1) had CpG island in the promoter region and six (adh1, SPBC1773, SPCC13B11.04c, SPAC2E1P3.01, Yak3, and SPBC16A3.02c) CpG islands

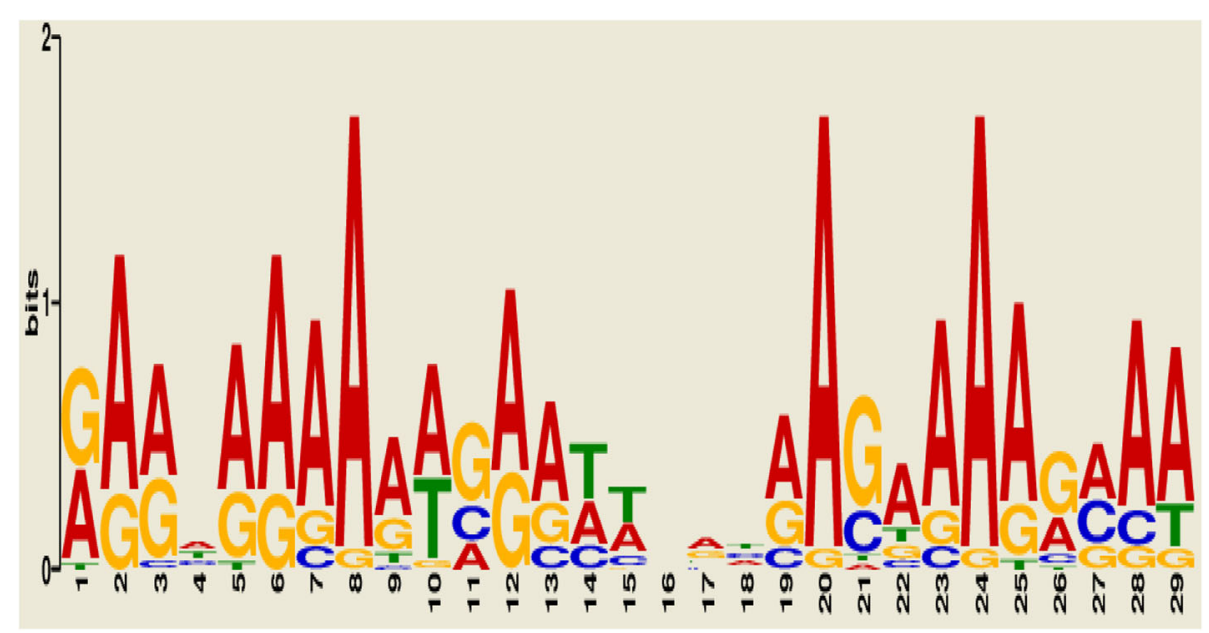

Fig. 2 Sequence logo for the identified common promoter motif MSp1 for gene encoding alcohol production gene in S. pombe 


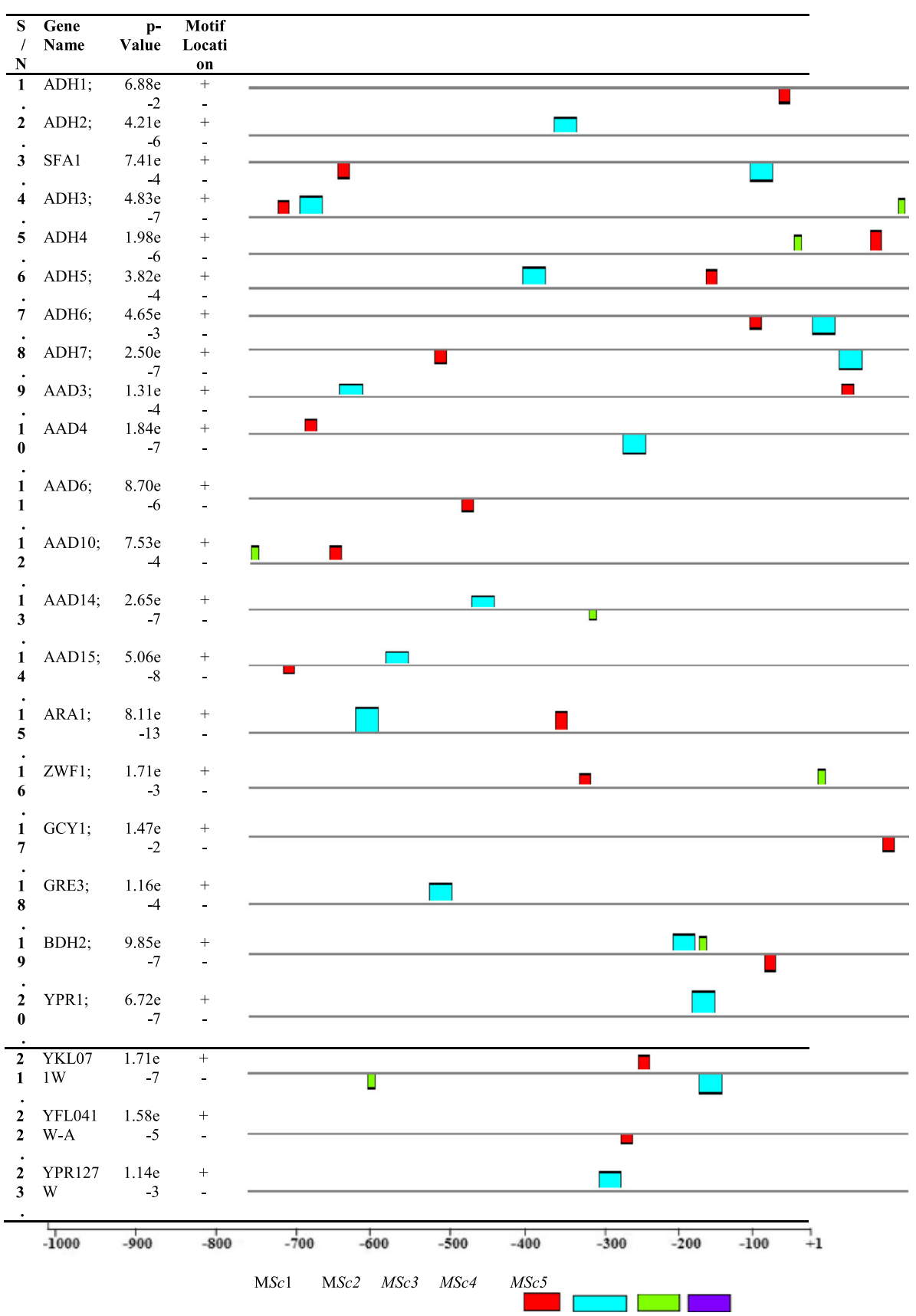

Fig. 3 The relative positions of motifs in alcohol dehydrogenase for S. cerevisiaea S288C sequences relative to TSSS

were observed in the gene body of genes encoding for alcohol production of S. pombe $972 \mathrm{~h}$.

On the other hand, CLC genomics workbench ver 3.6.1 using restriction enzyme MSpI (C/CGG sequence) cutting sites with standard fragment sizes between 40 and $220 \mathrm{bp}$ revealed that in S. cerevisiaea $S 288 C$ five (ARA1, BDH2, GCY1, SFA1, and GRE3) and only one (GRE3) CpG islands were found in gene body and promoter regions, respectively. In contrarily, the number of CpG islands in the gene body was only one (SPBC16A3.02c) while four (adh1
(Fig. 6), adh4, SPAP32A8.02, and SPBC337.11) in the promoter regions of $S$. pombe $972 h$ - in alcohol dehydrogenase (Table 5). This indicates the poor occurrence of CpG islands in both gene body and promoter regions which may affect the access of promoter region of genes to their transcription factors, hence preventing their expression.

\section{Phylogenetic analysis}

A combined analysis of all the data weighted equally resulted in a single most-parsimonious cladogram (Fig. 7). 


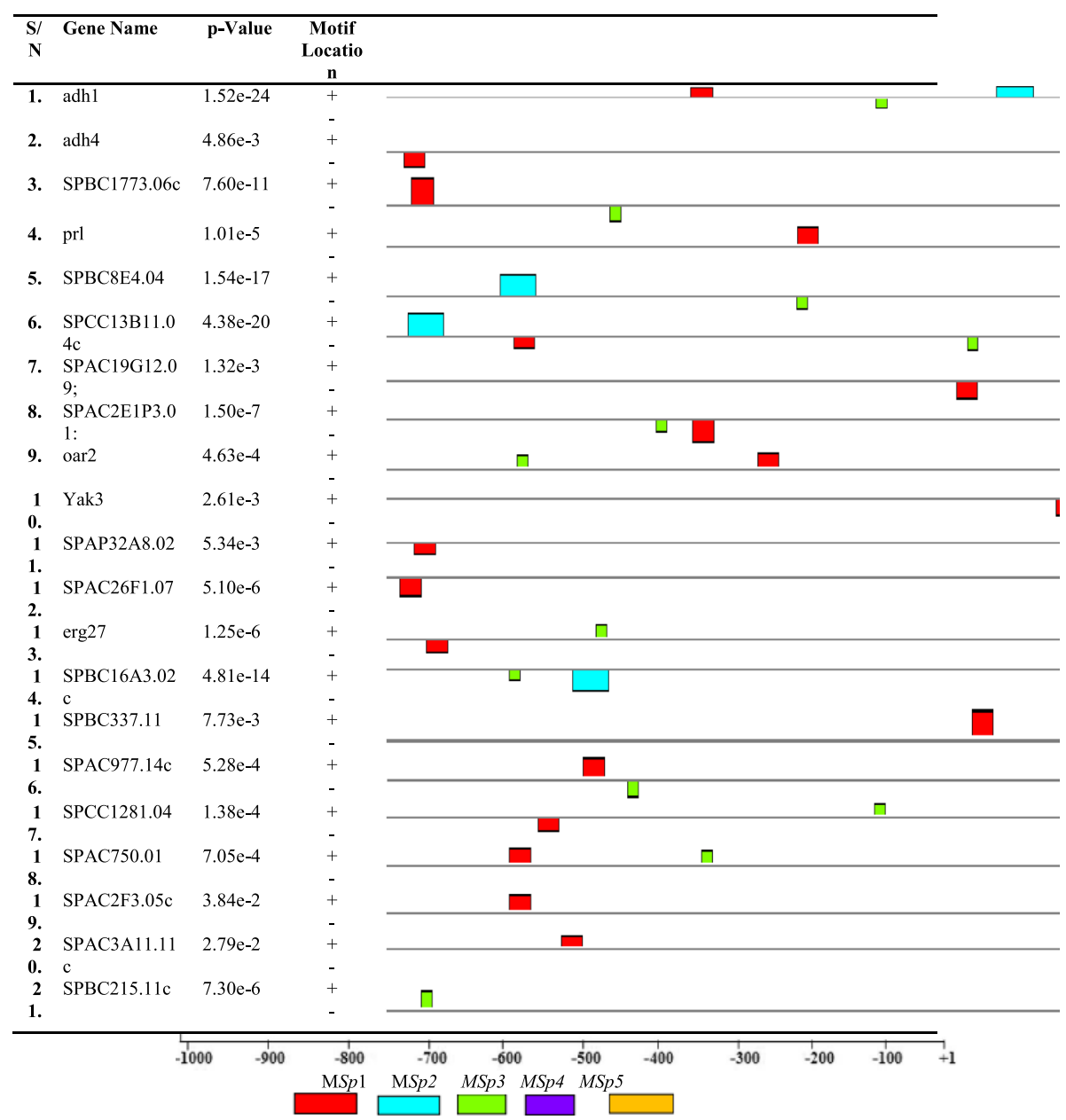

Fig. 4 The positions and strand orientation of motifs of S. pombe alcohol production gene relative to TSSS

Statistics for this tree revealed that in general, relationships on the tree are very strongly supported. A phylogenetic tree was generated using the neighbor-joining (NJ) as well as minimum-evolution method of MEGA 6.0. As illustrated in Fig. 7, all sequences from both $S$. cerevisiaea $S 288 \mathrm{C}$ and S. pombe $972 \mathrm{~h}$ - were divided into four subgroups (I, II, III, and IV). The four clades are respectively colored in blue, red, green, and violet. The phylogenetic tree indicated that some gene sequences irrespective of source organism clustered together within each sub-group suggest a close evolutionary relationship among the genes rather than the whole species.

\section{Discussion}

Sequence-specific DNA-binding transcription factors (TFs) are often termed as "master regulators" which bind to DNA and either activate or repress gene transcription. Determination of a gene's transcriptional start site underlies the identification of the proximal promoter region and thus facilitates the subsequent analysis of gene expression. In the current in silico analysis, majority of the sequences in both species had multiple transcription start sites. This could give the alternative transcription potential for the gene sequence under consideration. However, for better prediction, TSS with a higher cutoff value was considered in the current study. Many authors [22-24] have reported the presence of multiple transcription sites in genes encoding for alcohol production. The comparison of the two yeast species with this regard showed that $S$. cerevisiaea $S 288 \mathrm{C}$ had a nearby site than S. pombe $972 h$ - This may be due to the fact that $S$. cerevisiaea $S 288 \mathrm{C}$ heavily relies on genes encoding for alcohol production to convert aldehydes and ketones into alcohols and $\mathrm{NADH}$ to $\mathrm{NAD}^{+}$that the yeasts can use for energy [25]. This process of yeasts turning aldehydes and ketones into alcohols is called fermentation. In general, promoter regions are located at the immediately upstream of a transcription start site (TSS) and have a variety of sequence motifs that participate in gene regulation [26].

The establishment and maintenance of temporal and spatial patterns of gene expression are achieved primarily 

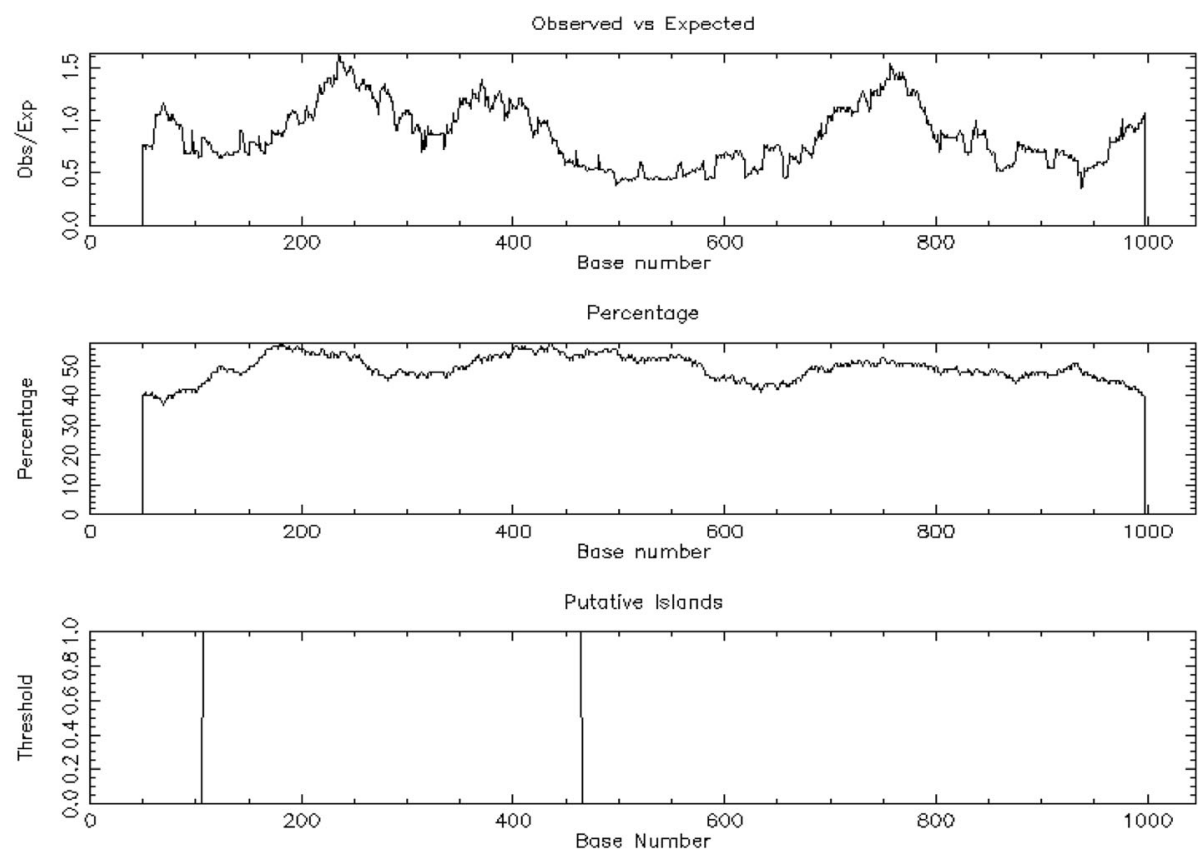

Fig. 5 CPGPLOT islands of ADH1, with length 358 (108-465)
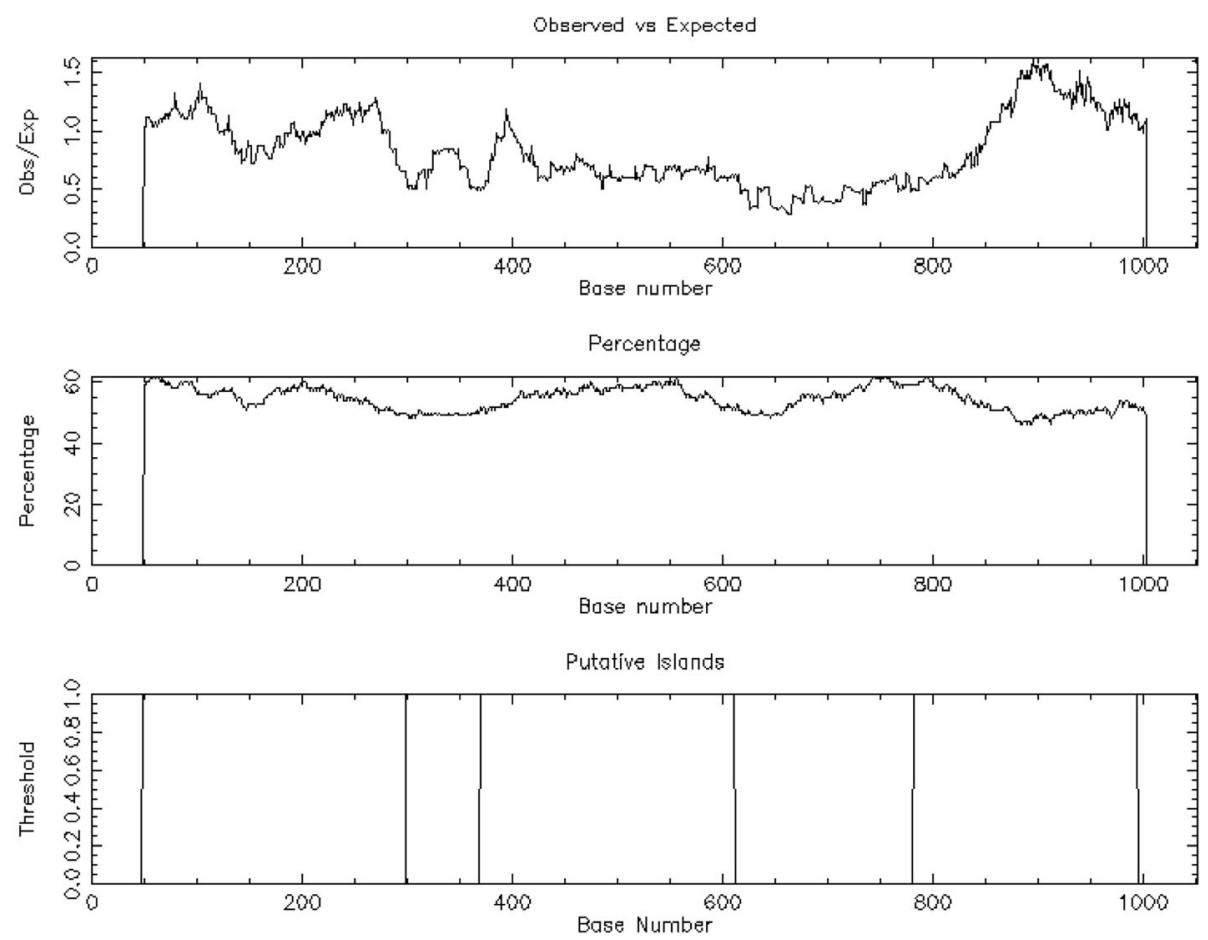

Fig. 6 CPGPLOT islands of three regions of adh1 gene; from 1 to 1053 with length 251 (49-299), length 243 


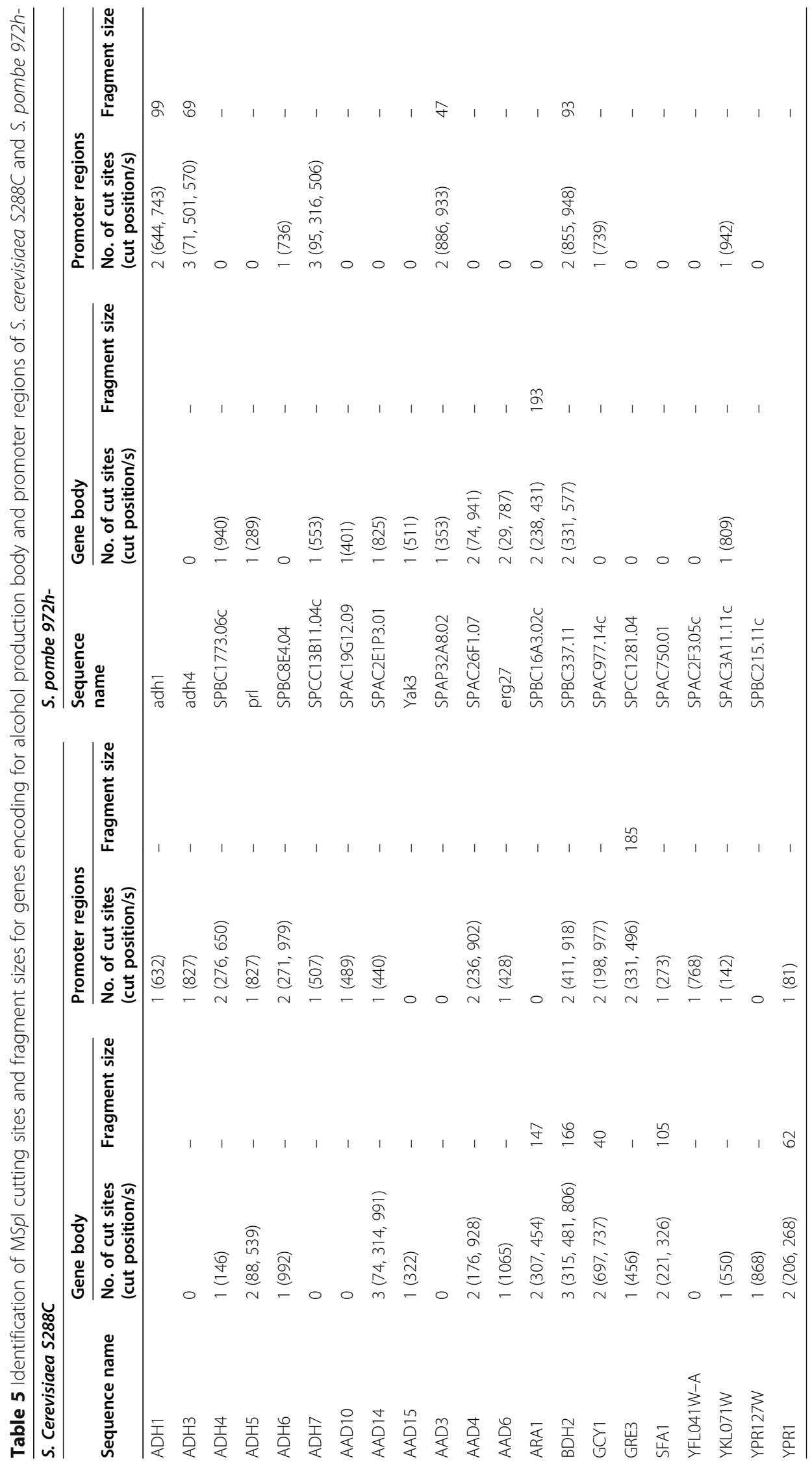




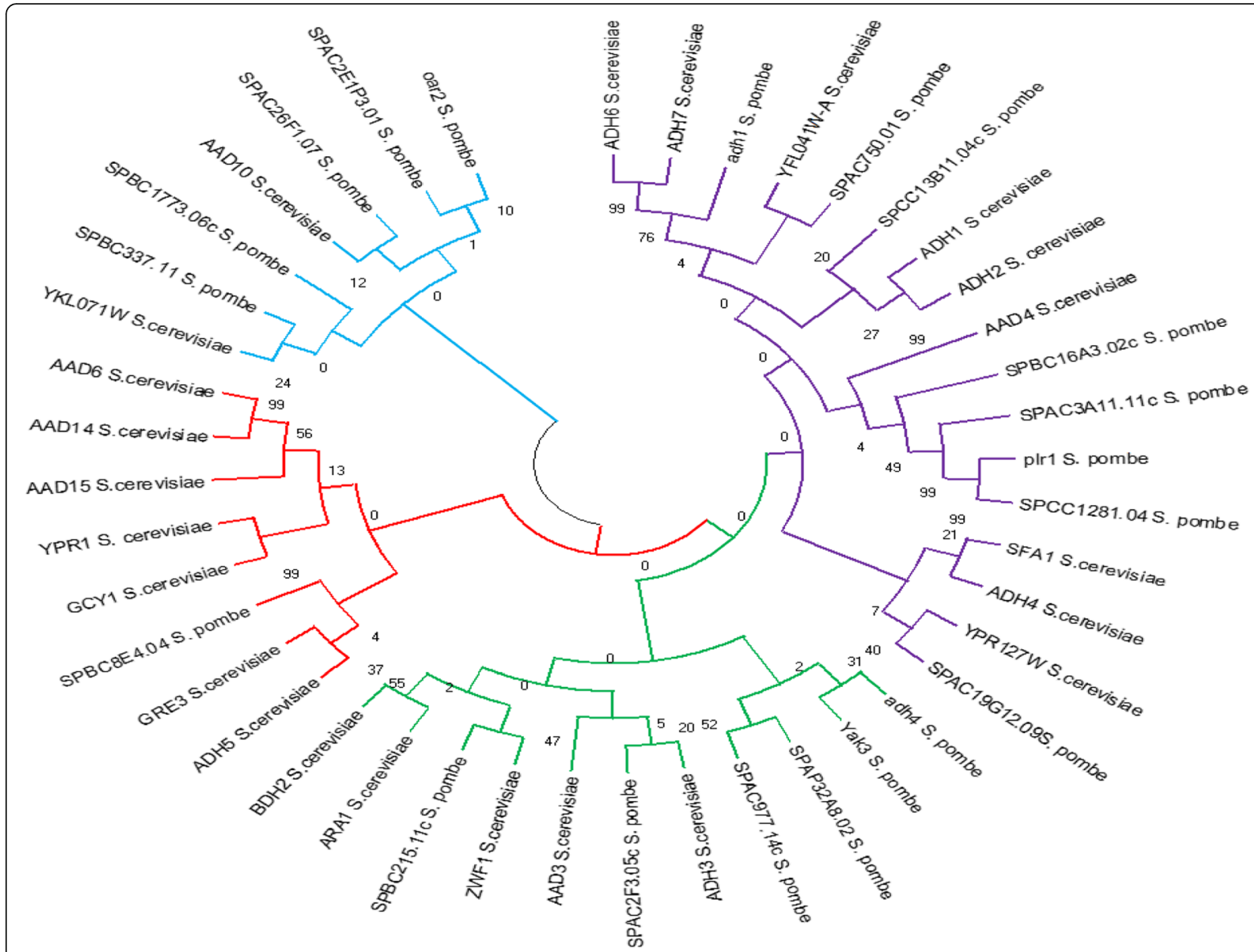

Fig. 7 Phylogenetic tree of genes encoding alcohol production sequence from S. cerevisiaea S288C and S. pombe 972h-

by transcription regulation. Functionally important motifs are usually short conserved sequence pattern associated with distinct functions of DNA often serve as transcription factor biding sites. In the current in silico analysis using MEME, the conserved short sequences of S. cerevisiaea S288C had confirmed to have higher occurrencesthan that of S. pombe 972h-. Common motifs, short DNA segments, are binding sites for transcription factors [27]. In both cases, the probability of finding a well-conserved pattern in random sequences as evidenced by an $E$ value is significantly higher than expected value. It is generally believed that genes having similar expression patterns contain common motifs in their promoter regions [28, 29]. Common promoter motifs are the key signatures for a family of coregulated genes and are usually present in the regions where complex protein interactions occur [30]. However, in some cases, single motifs can bind various transcription factors thereby bringing the genes under multiple regulatory controls [31, 32]. Extensive studies on 500 bp upstream regions of yeast promoters suggest that regulatory elements are commonly present in those regions [33].
It is well reported that CGIs are highly involved in gene regulatory processes [34]. They are present at or near the gene's transcription start site and are often associated with the promoters of most house-keeping genes and many tissue-specific genes, and thus have important regulatory functions and can be used as gene markers [35].

A phylogenetic tree generated in the current study showed that all sequences from both $S$. cerevisiaea S288C and S. pombe $972 h$ - were divided into four subgroups. This could be due to the fact that some vital genes for alcohol fermentation of yeast strains. Similar studies by other authors [34, 35] have revealed that one organism which is equally amenable to genetic manipulation as is $S$. cerevisiaea is the fission yeast S. pombe. Although the two organisms are similar in that they are ascospore-forming yeasts capable of strong alcoholic fermentation, S. pombe actually has diverged significantly from $S$. cerevisiaea [36, 37]. In fact, the available information suggests that $S$. pombe may actually be more closely related to filamentous fungi such as Neurospora and Asperglus than it is to budding yeast $[9,38]$. 


\section{Conclusion}

The enhancement of DNA transformation in yeast has resulted in revolutionized molecular tools and facilitated ease of thought of aspects of gene structure and regulation of gene expression of many genes from baker's yeast (S. cerevisiaea) and fission yeast (S. pombe). Generally, the regulation of alcohol dehydrogenase enzyme which is critically important for the survival and enhanced efficiency of yeast species can better be examined and explained with the use of increasing technological advancement, genome mining, and in silico analysis of gene sequences. Majority of the genes encoding for alcohol production sequences have multiple TSS in both yeast species suggesting alternative gene regulation.

The result of this analysis could be critically important to understand the nature of promoter regions, the motif discovered in line with the transcription factor binding proteins, and the strength of the genome to different transcriptions via following the frequency of CpG islands. The phylogenetic analysis revealed that majority of genes is clustered together irrespective of the source organism suggesting a close evolutionary relationship among the gene rather than the whole species.

In general, this in silico analysis of genes encoding for alcohol production of S. cerevisiaea $S 288 C$ and S. pombe could be helpful to add knowledge about the species molecular data and supportive to identify gene regulatory elements in the promoter regions. It could also help to predict gene expression profiles in various yeast species which in turn could be helpful to improve efficiencies of these organisms for domestic and commercial production of bio-fuel with higher rate of recovery.

\section{Abbreviations \\ TSS: Transcription start siteMSC1Motif of Saccharomyces cerevisiaea 1 MSp1Motif of Schizosaccharomyces pombes. cerevisiaeaSaccharomyces cerevisiaeaS. pombeSchizosaccharomyces pombeTFsTranscription factorsMEMEMultiple Em for Motif ElicitationNCBINational Center for Biotechnology InformationbpBase pairNNPPNeural Network Promoter Prediction}

\section{Acknowledgements}

The authors acknowledge Adama Science and Technology University, School of Applied Natural Science, for funding the research.

\section{Authors' contributions}

JA designed and performed the experiment, analyzed the data, and prepared the draft manuscript. MK designed the experiment, supervised the research, revised the manuscript, and is the correspondence of the paper. The authors read and approved the final manuscript.

\section{Funding}

This work was financially supported by the graduate program of Adama Science and Technology University.

\section{Availability of data and materials}

The qualitative and quantitative data of this manuscript are available through the first author.

Ethics approval and consent to participate Not applicable

\section{Consent for publication}

Not applicable

\section{Competing interests}

The authors declared that they have no competing interests.

Received: 19 June 2020 Accepted: 17 November 2020

Published online: 11 January 2021

\section{References}

1. P.Hazell, "B ioenergy and agriculture: promises and challenges. The International Food Policy Research Institute (IFPRI)," Focus 14 • Brief 2 Of 12 - Report uploaded by Peter Hazell on February 2015.

2. Ngwenya TT (2012) An industrial perspective of factors affecting molasses fermentation by Saccharomyces cerevisiae. J Brew Distill 3(2). https://doi. org/10.5897/jbd12.002

3. Nissen TL, Kielland-Brandt MC, Nielsen J, Villadsen J (2000) Optimization of ethanol production in Saccharomyces cerevisiae by metabolic engineering of the ammonium assimilation. Metab Eng 2(1):69-77. https://doi.org/10. 1006/mben.1999.0140

4. V. Ansanay-galeote, B. Blondin, S. Dequin, and J. Sablayrolles, "Stress effect of ethanol on fermentation kinetics by stationary-phase cells of Saccharomyces cerevisiae Stress effect of ethanol on fermentation kinetics by stationary-phase cells of Saccharomyces cerevisiae," 2001, 10.1023/A.

5. Fadel M, Keera AA, Mouafi FE, Kahil T (2013) High Level Ethanol from Sugar Cane Molasses by a New Thermotolerant Saccharomyces cerevisiae Strain in Industrial Scale. Biotechnol Res Int 2013:1-6. https://doi.org/10.1155/2013/ 253286

6. Stewart GG, Panchal C, Russell I, Sills AM (2008) Biology of EthanolProducing Microorganisms. Crit Rev Biotechnol 1(3):161-188. https://doi.org/ 10.3109/07388558309077977

7. Russell BDHPR (1983) The primary structure of the alcohol dehydrogenase gene from the fission yeast Schizosaccharomyces pombe. J Biol Chem 10(1): $143-149$

8. Weber T (2014) In silico tools for the analysis of antibiotic biosynthetic pathways. Int J Med Microbiol 304(3-4):230-235. https://doi.org/10.1016/j. ijmm.2014.02.001

9. Ziemert N, Alanjary M, Weber T (2016) The evolution of genome mining in microbes-a review. Nat Prod Rep 33(8):988-1005. https://doi.org/10.1039/ c6np00025h

10. González C, Salces-Ortiz J, Calvo JH, Serrano MM (2016) In silico analysis of regulatory and structural motifs of the ovine HSP90AA1 gene. Cell Stress Chaperones 21(3):415-427. https://doi.org/10.1007/s12192-016-0668-6

11. Peirce BA (2010) Genetics: A Conceptual Approach, 4th edn. W. H. Freeman ISBN-10 : 1429232501

12. Abeel T, Saeys Y, Rouzé P, Van De Peer Y (2008) ProSOM : core promoter prediction based on unsupervised clustering of DNA physical profiles. 24 : 24-31. https://doi.org/10.1093/bioinformatics/btn172

13. Korkuć P, Schippers JHM, Walther D (2014) Characterization and identification of cis-regulatory elements in arabidopsis based on singlenucleotide polymorphism information. Plant Physiol 164(1):181-200. https:// doi.org/10.1104/pp.113.229716

14. Borneman AR, Gianoulis TA, Zhang ZD (2007) Divergence of Transcription Factor Binding Sites Across Related Yeast Species. Science 317:815-820 www.sciencemag.org

15. Robertson KD (2002) DNA methylation and chromatin - Unraveling the tangled web. Oncogene 21(35 REV. ISS. 3):5361-5379. https://doi.org/10. 1038/sj.onc.1205609

16. Du X, Han L, Guo AY, Zhao Z (2012) Features of methylation and gene expression in the promoter-associated CpG islands using human methylome data. Comp Funct Genomics 2012. https://doi.org/10.1155/ 2012/598987

17. Gupta S, Stamatoyannopoulos JA, Bailey TL, Noble WS (2007) Quantifying similarity between motifs. 8(2). https://doi.org/10.1186/gb-2007-8-2-r24

18. Reese MG (2001) Application of a time-delay neural network to promoter annotation in the Drosophila melanogaster genome. Comput Chem 26:5156

19. Bailey TL et al (2009) MEME S UITE : tools for motif discovery and searching: 1-7. https://doi.org/10.1093/nar/gkp335

20. Takai D, Jones PA (2002) Comprehensive analysis of CpG islands in human chromosomes 21 and 22. 99(6):3740-3745 
21. Sandelin A, Alkema W, Lenhard B (2004) JASPAR : an open-access database for eukaryotic transcription factor binding pro ${ }^{\circledR}$ les. 32 (iii). https://doi.org/10. 1093/nar/gkh012

22. Irani M, Taylor WE, Young ET (1987) Transcription of the ADH2 gene in Saccharomyces cerevisiae is limited by positive factors that bind competitively to its intact promoter region on multicopy plasmids. Mol Cell Biol 7(3):1233-1241. https://doi.org/10.1128/mcb.7.3.1233

23. Ishida Y, Nguyen TTM, Izawa S (2017) The yeast ADH7 promoter enables gene expression under pronounced translation repression caused by the combined stress of vanillin, furfural, and 5-hydroxymethylfurfural. J Biotechnol 252:65-72. https://doi.org/10.1016/j.jbiotec.2017.04.024

24. Mohanty B, Takahashi H, de los Reyes BG, Wijaya E, Nakazono M, Lee DY (2016) Transcriptional regulatory mechanism of alcohol dehydrogenase 1deficient mutant of rice for cell survival under complete submergence. Rice 9(1):1-8. https://doi.org/10.1186/s12284-016-0124-3

25. De Smidt O, Du Preez JC, Albertyn J (2008) The alcohol dehydrogenases of Saccharomyces cerevisiae: A comprehensive review. FEMS Yeast Res 8(7): 967-978. https://doi.org/10.1111/j.1567-1364.2008.00387.x

26. Halees AS, Leyfer D, Weng Z (2003) PromoSer: A large-scale mammalian promoter and transcription start site identification service. Nucleic Acids Res 31(13):3554-3559. https://doi.org/10.1093/nar/gkg549

27. Das MK, Dai H (2007) A survey of DNA motif finding algorithms. 13:1-13. https://doi.org/10.1186/1471-2105-8-S7-S21

28. Brazma A, Bergen N, Robinson A (1998) Using Gene Expression Data. 17: 279-287

29. Klok EJ et al (2002) Expression profile analysis of the low-oxygen response in arabidopsis root cultures. Plant Cell 14(10):2481-2494. https://doi.org/10. 1105/tpc.004747

30. Wang Z, Dalkilic M, Kim S (2004) Guiding motif discovery by iterative pattern refinement. Proc ACM Symp Appl Comput 1:162-166. https://doi. org/10.1145/967900.967934

31. Jin H, Martin C (1999) Multifunctionality and diversity within the plant MYBgene family. Plant Mol Biol 41(5):577-585. https://doi.org/10.1023/A: 1006319732410

32. Yanagisawa S (2002) The Dof family of plant transcription factors. Trends Plant Sci 7(12):555-560. https://doi.org/10.1016/S1360-1385(02)02362-2

33. Caselle M, Di Cunto F, Provero P (2002) Correlating overrepresented upstream motifs to gene expression : a computational approach to regulatory element discovery in in eukaryotes. BMC Bioinformatics 3:1-10

34. Deaton AM, Bird A (2011) CpG islands and the regulation of transcription. Genes Dev 25(10):1010-1022. https://doi.org/10.1101/gad.2037511

35. Ye S, Asaithambi A, Liu Y (2008) CpGIF: an algorithm for the identification of CpG islands. Bioinformation 2(8):335-338. https://doi.org/10.6026/ 97320630002335

36. Riveros-Rosas H, Julián-Sánchez A, Villalobos-Molina R, Pardo JP, Piña E (2003) Diversity, taxonomy and evolution of medium-chain dehydrogenase/ reductase superfamily. Eur J Biochem 270(16):3309-3334. https://doi.org/10. 1046/.1432-1033.2003.03704.x

37. Kuramae EE, Robert V, Snel B, Boekhout T (2006) Conflicting phylogenetic position of Schizosaccharomyces pombe. 88:387-393. https://doi.org/10. 1016/j.ygeno.2006.07.001

38. Liu Q, Wang X (2020) Characterization and phylogenetic analysis of the complete mitochondrial genome of a basidiomycetous yeast Cystobasidium sp. (Cystobasidiales: Cystobasidiaceae). Mitochondrial DNA Part B Resour 5(3):2449-2450. https://doi.org/10.1080/23802359.2020.1777910

\section{Publisher's Note}

Springer Nature remains neutral with regard to jurisdictional claims in published maps and institutional affiliations.

\section{Submit your manuscript to a SpringerOpen ${ }^{\circ}$ journal and benefit from:}

- Convenient online submission

- Rigorous peer review

- Open access: articles freely available online

High visibility within the field

- Retaining the copyright to your article

Submit your next manuscript at $\boldsymbol{\nabla}$ springeropen.com 